\title{
A study of maternal and fetal outcome in third trimester diagnose case of oligohydramnios
}

\author{
Vibha Moses*, Sarita Thakre
}

Department of Obstetrics and Gynaecology, MGM Medical College, Indore, Madhya Pradesh, India

Received: 14 August 2016

Accepted: 20 August 2016

\section{*Correspondence:}

Dr. Vibha Moses,

E-mail: drvibs2815@gmail.com

Copyright: ( $)$ the author(s), publisher and licensee Medip Academy. This is an open-access article distributed under the terms of the Creative Commons Attribution Non-Commercial License, which permits unrestricted non-commercial use, distribution, and reproduction in any medium, provided the original work is properly cited.

\section{ABSTRACT}

Background: Oligohydramnios is defined as when the maximum vertical pockets of liquor is less than $2 \mathrm{~cm}$ or when AFI is less than $5 \mathrm{~cm}$ or less than 10th centile. Amniotic fluid is part of the baby" life support system during labour so the purpose of this study is evaluate maternal and perinatal outcome in present study.

Methods: 150 patients with third trimester diagnosed case of oligohydramnios were included and were screened through exclusion and inclusion criteria.

Results: Oligohydramnios was more in primipara in our study. It was increase in case of prolonged pregnancy. 66\% were of moderate AFI and 29\% were severe oligohydramnios. Idiopathic was most common cause, and second cause was PIH. Oligohydramnios was related to higher rate of IUGR and NICU admission.

Conclusions: Now a day oligohydramnios is most common occurrence in pregnant women. Amniotic fluid volume is a predictor of fetal tolerance in labour and its decrease is associated with increased perinatal morbidity and mortality, rates of caesarean section are rising. Take timely intervention can reduce perinatal morbidity and mortality. Vaginal delivery and caesarean section should be well balanced so that unnecessary maternal morbidities prevented and improve labour outcome.

Keywords: Oligohydramnios, AFI perinatal morbidities, Maternal outcome

\section{INTRODUCTION}

Oligohydramnios is defined as when the maximum vertical pockets of liquor is less than $2 \mathrm{~cm}$ or when AFI is less than $5 \mathrm{~cm}$ or $10^{\text {th }}$ centile. ${ }^{1-3}$ Amniotic fluid is part of the baby" life support system during labour. ${ }^{2}$ The most important mechanical function of amniotic fluid is to provide an adequate cushion for the umbilical cord. Without this cushion, compression of the cord between the fetus and the uterine wall may occur during contractions or fetal movement. This cord compression lead to severe FHR decelerations which are associated with low APGAR scores and acidosis at birth, meconium staining, cesarean section and operative vaginal delivery for fetal distress
Oligohydramnios is also associated with maternal morbidity in form of increased rates of induction and/ or operative interference. Amniotic fluid index" was described by Phelan et al. $^{1-3}$ It is the most accurate method for assessing amniotic fluid volume and helps to categorize the patients into normal, low normal and oligohydramnios groups. Increased induction of labour and elective caesarean deliveries are currently practiced for better perinatal outcome. Early detection of oligohydramnios and its management may help in reduction of perinatal morbidity and mortality one side and decreased caesarean deliveries on the other side. Since oligohydramnios has got significant impact on perinatal outcome and maternal morbidity, oligohydramnios is a severe and common complication of 
pregnancy and the incidence of this is reported to be $3.9 \%$ of total pregnancies. Oligohydramnios in the third trimester of pregnancy has been associated with intrauterine growth restriction, post-dated pregnancy, congenital anomalies, increased fetal morbidity and abnormal antepartum fetal heart rate pattern, it is prompted us to study the maternal and perinatal outcome third trimester diagnosed case of oligohydramnios. ${ }^{4}$

\section{METHODS}

The present study was conducted in the Department of Obstetrics \& Gynaecology, M.G.M. Medical College Indore (M.P.), India during the period from February 2014-March 2015, 150 cases of oligohydramnios in third trimester selected after satisfying inclusion and exclusion criteria.

This study was observational and prospective study. Details of these patients were recorded in the proforma.

\section{Inclusion criteria}

USG proven cases of oligohydramnios, AFI $5 \mathrm{~cm}$, antenatal patient in third trimester, singleton pregnancy.

\section{Exclusion criteria}

AFI $>6 \mathrm{~cm}$, multiple gestation, patients having major respiratory, heart disease.

\section{Methodology}

Plan of activity and time chart were formulated after taking informed consent from the woman and/or relatives. Other potential explanatory variables were obtained including maternal age, booking status, PIH and other risk factors at the time of admission were recorded. Detailed clinical history including obstetric, menstrual, past and personal history were taken. Thorough general, systemic and obstetric examination was conducted. Woman's hematological profile was done. AFI to be measured using phelan's four quadrant ultrasound technique. ${ }^{1-3}$ The uterus is arbitrarily divided into four quadrants by the umbilicus transversely and the linea nigra vertically.

The largest vertical pocket free of fetal parts and umbilical cord loops in each quadrant is measured and sum of these measurements will give AFI in cm. An AFI of $5-24 \mathrm{~cm}$ is normal. AFI of $<5 \mathrm{~cm}$ is considered Oligohydramnios. USG at the time of admission was recorded including fetal biometry, amniotic fluid volume and color doppler. Study of association of various maternal factors like prolong pregnancy, hypertensive disorders of pregnancy, PROM etc. Outcome was noted in the form of mode of delivery, fetal outcome, apgar score, fetal birth weight, maturity, admission to nursery and postnatal complications, if any.

\section{RESULTS}

All the information was entered in the proforma and analyzed and observations were made and accordingly discussion. $58 \%$ of patients were in 20-25 years age group and $29 \%$ in $26-30$ year age group thus maximum patient were in 26-30 year.Mean meternal age was23.66 (Table 1) .Table 2 shows that primipara patients form a major burden of admissions due to oligohydramnios. This table 3 shows that case of oligohydramnios according to gestational age. It was increase in case of prolonged pregnancy.

Mode of Delivery: $42 \%$ cases were delivered by LSCS (Table 6).

Table 1: Distribution of patients according to age.

\begin{tabular}{|lll|}
\hline Age (Years) & Oligohydramnios \\
\hline $18-20$ & No. & \% \\
\hline $20-25$ & 11 & $07 \%$ \\
\hline $26-30$ & 87 & $58 \%$ \\
\hline$>30$ & 43 & $29 \%$ \\
\hline Total & 09 & $06 \%$ \\
\hline
\end{tabular}

Table 2: Distribution of patients according to parity.

\begin{tabular}{|lll|}
\hline \multirow{2}{*}{ Parity } & \multicolumn{2}{c|}{ Cases $\mathbf{1 5 0}$} \\
\hline Para 0 & No. & \% \\
\hline Para 1 & 44 & 29 \\
\hline Para 2 & 85 & 57 \\
\hline Para 3 and above & 15 & 10 \\
\hline
\end{tabular}

Table 3: According to gestational age.

\begin{tabular}{|lll|}
\hline \multirow{2}{*}{ Gestational age (weeks) } & \multicolumn{2}{l|}{ Cases $\mathbf{1 5 0}$} \\
\hline $28-32$ & No. & $\%$ \\
\hline $33-34$ & 21 & $14 \%$ \\
\hline $35-37$ & 33 & $22 \%$ \\
\hline $38-40$ & 33 & $22 \%$ \\
\hline$>41$ & 28 & $19 \%$ \\
\hline
\end{tabular}

AFI wise distribution; in the present study $66 \%$ were of moderate AFI and $29 \%$ were severe oligohydramnios and $5 \%$ anhydramnios (Table 4 ).

Table 4: Classification of patients according to AFI.

\begin{tabular}{|c|c|c|}
\hline \multirow{2}{*}{ AFI (up to $5 \mathrm{~cm}$ ) } & \multicolumn{2}{|c|}{ Cases 150} \\
\hline & No. & $\%$ \\
\hline $3.1-5$ & 99 & 66 \\
\hline $1-3$ & 43 & 29 \\
\hline Anhydramnios & 08 & 05 \\
\hline
\end{tabular}


Table 5: Maternal factors associated with oligohydramnios.

\begin{tabular}{|lll|}
\hline Maternal Factors & $\begin{array}{l}\text { Total } \\
\text { No. }\end{array}$ & $\%$ \\
\hline & & \\
Prolonged pregnancy (>40wks) & 35 & 23 \\
\hline PIH & 40 & 26 \\
Gestational Hypertension & 23 & \\
Preeclampsia & 13 & \\
Eclampsia & 04 & \\
\hline PROM & 14 & 9 \\
\hline Idiopathic & 48 & 32 \\
\hline malpresentation & 05 & 3 \\
\hline Chorioamnionitis & 0 & 0 \\
\hline Chronic renal disease & 02 & \multicolumn{2}{c|}{1.4} \\
\hline Chronic abruptio & 06 & 4 \\
\hline
\end{tabular}

Table 6: Distribution of cases according to mode of delivery.

\begin{tabular}{|c|c|c|}
\hline \multirow{2}{*}{ Mode of Delivery } & \multicolumn{2}{|c|}{ Cases $(\mathbf{N}=150)$} \\
\hline & No. & $\%$ \\
\hline LSCS & 64 & 42 \\
\hline Vaginal Delivery & 86 & 57 \\
\hline
\end{tabular}

Table 7: Indication for LSCS.

\begin{tabular}{|lll|}
\hline \multirow{2}{*}{ Indication for LSCS } & Cases \\
\hline Total LSCS & No. & $\%$ \\
\hline Fetal distress & 64 & 42 \\
\hline MSL & 09 & 14 \\
\hline Anhydramnios & 08 & 12.5 \\
\hline Malpresentation (breech) & 05 & 7.8 \\
\hline Failed induction & 07 & 7.9 \\
\hline Previous section & 07 & 7.9 \\
\hline Oligohydramnios/IUGR with & 08 & 12.5 \\
\hline Doppler changes & 21 & 32.8 \\
\hline Chorioamniotis & 01 & 1.5 \\
\hline Cord around Neck & 01 & 1.5 \\
\hline Bad obstetric history & 01 & 1.5 \\
\hline
\end{tabular}

Table 8: Distribution of apgar score at birth at 1 min and $5 \mathrm{~min}$.

\begin{tabular}{|lll|}
\hline Apgar Score & At 1 min & At 5 min \\
\hline $8-10$ & 4 & 67 \\
\hline $5-7$ & 126 & 66 \\
\hline $3-4$ & 14 & 08 \\
\hline$<3$ & 1 & 0 \\
\hline
\end{tabular}

It was seen that LSCS in the study group was primarily done for oligohydramnios/IUGR, fetal hypoxia or fetal distress/meconium stained liquor (Table 7).

\section{Perinatal outcome}

In the present study, the Apgar score was noted 1 and 5 minutes after birth (Table 8).

Table 9: Distribution of cases according to perinatal outcome.

\begin{tabular}{|c|c|c|}
\hline \multirow{2}{*}{ Perinatal Outcome } & \multicolumn{2}{|c|}{ Cases } \\
\hline & No. & $\%$ \\
\hline Nursery admission & 52 & 35 \\
\hline Alive and healthy & 93 & 62 \\
\hline Still birth & 05 & 3.33 \\
\hline FSB & 04 & 2.67 \\
\hline MS & 01 & 0.67 \\
\hline
\end{tabular}

Table 10: Distribution of cases according to perinatal mortality.

\begin{tabular}{|lll|}
\hline \multirow{2}{*}{ Perinatal Mortality } & Cases \\
\hline Still birth & No. & $\%$ \\
\hline FSB & 05 & 3.33 \\
MSB & 04 & 2.67 \\
\hline Early neonatal death & 01 & 0.67 \\
\hline Total perinatal mortality & 15 & 10 \\
\hline
\end{tabular}

\section{DISCUSSION}

Perinatal morbidity and mortality are significantly increased when oligohydramnios is present. Accurate antepartum estimation of amniotic fluid volume by clinical means alone is difficult but it is easily diagnosed by current ultrasound methods. ${ }^{1-3}$ With the easier availability of ultrasonography nowadays more cases of oligohydramnios are being identified. This helps us to be more cautious and anticipate problems especially during labour. In the present study $58 \%$ of patients were in 20 25 years age group and $29 \%$ in 26-30 year age. Mean meternal age was 23.66. Similar studies by Chauhan P et al found that the mean maternal age were $23.6 \pm 6.5$ years. In Donald $\mathrm{D}$ et el the incidence of oligohydramnios was $60 \%$ in primigravida which is comparable to present study $57 \%$ patients were para $1 .^{5,6}$ According to gestational age it is increase in $>40$ weeks pregnancy $23 \%$ in the present study. Clement D et al studied six cases of postdatism, in which amniotic fluid volume diminished abruptly over 24 hours. ${ }^{7}$ Bowen Chattoor JS et al in their study evaluated the relationship between amniotic fluid index and perinatal outcomes in fifty five postdate pregnancies. ${ }^{17}$

Obstetrical complications frequently associated with oligohydramnios .in present study idiopathic cause $32 \%$, PIH was present in $25 \%$ cases. Similar result found in Golan A et al in his study, found maternal hypertension in $22.1 \%$ cases. $^{8}$ Cesarean section was performed in $32.8 \%$ of these cases. Mercer $\mathrm{LJ}$ et al found that preeclampsia was present in $24.7 \%$ of cases with decreased fluid. Study by Chauhan $\mathrm{P}$ et al. reported, 
preeclampsia in $12 \%$ cases. $^{5}$ They concluded that the incidence of oligohydramnios ranges from 10 to $30 \%$ in hypertensive patients requiring hospitalization.

\section{Mode of delivery}

The LSCS was done in $42 \%$ in present study which is compared with the situations in other studies. Study by Casey B et al found that, there was increased rate of induction of labour (42\%) and cesarean section (32\%) in oligohydramnios cases. ${ }^{9}$ Golan A et al. found that, the cesarean section was performed in $35.2 \%$ of pregnancies. ${ }^{8}$ These are comparable to my study.

\section{Perinatal outcome}

In the present study, the Apgar score was noted at 1 and 5 minutes after birth. 16.3\% babies had low Apgar score (less than 7 at 1 and $5 \mathrm{~min}$ ). Syria $\mathrm{R}$ et al in their study have reported $38.8 \%$ incidence of Apgar score less than 7 at 1 minute. ${ }^{10}$ In a similar study by Casey B et al $(6 \%)$ babies had Apgar score of less than 3 at 5 minute. ${ }^{9}$ Out of these nine babies, seven died during neonatal period. Jun Zhang et al found that an Apgar score of $<7$ at 1 minute was present in fifteen. ${ }^{12}$ Six babies had Apgar score of $<7$ at 5 minute.

Notably; the incidence of meconium aspiration syndrome in infants with oligohydramnios was significantly higher despite the diminished identification of meconium stained amniotic fluid. Bowen Chattoor JS et al studied perinatal outcome in 55 postdate pregnancies. Oligohydramnios was noted in four patients. ${ }^{16}$ All 4 babies were admitted with meconium aspiration. One died due to this complication.

In our study neonates of $35 \%$ cases were admitted in nursery. Syria $\mathrm{R}$ et al has reported a very high incidence of NICU admission. ${ }^{10}$ In their study $88.88 \%$ newborns were admitted in NICU in patients having $\mathrm{AFI}<5 \mathrm{~cm}$ Casey BM et al in their study have reported $7 \%$ admission to the NICU in patients with AFI $<5 \mathrm{~cm}$. Zhang $\mathrm{J}$ et al in their study have reported $29.4 \%$ admission to NICU in patients with AFI $<5 \mathrm{~cm}$.

In the present study, there were $62 \%$ live births and $3 \%$ still births. $10 \%$ babies died in neonatal period. The perinatal mortality was $13 \%$ in present study. Chamberlin PF et al. calculated the perinatal mortality rate in patients with decreased qualitative amniotic fluid volume and found it to be $188 / 1000$. Chhabra $S$ et al reported very high $(87.7 \%)$ perinatal mortality in their study. ${ }^{14}$ Wolff $\mathrm{F}$ et al found that the perinatal mortality in their study was $7.2 \% .^{15}$ Apel SL et al found that the perinatal mortality was $9.9 \% .^{17}$ The lack of amniotic fluid allows compression of fetal abdomen, which limits the movement of the diaphragm. Overall, the perinatal mortality is markedly increased in patients with oligohydramnios.

\section{CONCLUSION}

In presence of oligohydramnios thorough evaluation of the gravida for hypertension, pre-eclampsia, diabetes, chronic abruption, premature rupture of membrane, drug intake etc should be done. An amniotic fluid index of $\leq 5$ $\mathrm{cm}$ detected in third trimester was associated with adverse pregnancy out come as well as indicator of poor perinatal outcome.

Now a days oligohydramnios is most common occurrence in pregnant women. Amniotic fluid volume is a predictor of fetal tolerance in labour and its decrease is associated with increased perinatal morbidity and mortality, rates of cesarean section are rising. Take timely intervention can reduce perinatal morbidity and mortality. Regular antenatal and intranatal monitoring should be done to diagnose any fetal compromise at the earliest. Termination of pregnancy according to the balance of risk of intrauterine asphyxia against those of prematurity should be done to obtain the best outcome. Vaginal delivery and caesarean section should be well balanced so that unnecessary maternal morbidities prevented and improve labour outcome.

\section{ACKNOWLEDGEMENTS}

Authors would like to acknowledge the teachers and their colleagues, for providing an impetus to perform better.

Funding: No funding sources Conflict of interest: None declared

Ethical approval: The study was approved by the Institutional Ethics Committee

\section{REFERENCES}

1. Rutherford SE, Phelan JP, Smith CV, Jacobs N. The four-quadrant assessment of amniotic fluid volume: an adjunct to antepartum fetal heart rate testing. Obstet Gynecol. 1987;70(3):353-6.

2. Ali HS. Assessment of amniotic fluid index in normal pregnancy at a tertiary care hospital setting. Department of Obstetrics \& Gynaecology, Ziauddin University, Karachi, Pakistan. J Ayub Med Coll Abbottabad. 2009;21(3):149-50.

3. Moore TR. Clinical assessment of amniotic fluid. Clin Obstet Gynecol. Lippincott-Raven Publishers, 1997;40(2):302-13.

4. Callen. Amniotic fluid; its role in fetal health and disease In: Textbook of ultrasonography in obstetrics and gynecology. $14^{\text {th }}$ ed. W. B. Saunders company:2000;638-59.

5. Chauhan SP, Hendrix NW: Intrapartum oligohydramnios does not predict adverse peripartum outcome among high risk parturients Am J Obstet Gynecol. 1997;176(6):1130-6.

6. Donald DM. Pregnancy outcomes after antepartum diagnosis of oligohydramnios at or beyond 34 
weeks' of gestation. Am J Obstet Gynecol. 2000;182(4):909-12.

7. Clement D, Schifrin BS, Kates RB. Acute oiligohydramnios in postdated pregnancy. AM J Obstet Gynecol. 1987;157(4):884-6.

8. Golan G. Oligohydramnios-maternal complication and fetal outcome in 145 cases. Gynecol Obstet Invest. 1994;37(2):91-5.

9. Casey BM, McIntire DD, Bloom SL, Lucas MJ, Santos R, Twickler DM, et al. Pregnancy outcomes after antepartum diagnosis of oligohydramnios at or beyond 34 weeks' of gestation. Am J Obstet Gynecol 2000;182(4):909-12.

10. Sriya R, Singhai S. Perinatal outcome in patients with amniotic fluid index $<5 \mathrm{~cm}$. J Obstet Gynaecol India. 2001;51:98-100.

11. Umber A. Perinatal outcome in pregnancies complicated by isolated oligohydramnios at term. Annals. 2009;15:35-7.
12. Jun Z, Troendle J. Isolated oligohydramnios is not associated with adverse perinatal outcome. British $\mathbf{J}$ Obst Gynae. 2004;111(3):220-5.

13. Chandra P, Kaur SP, Hans DK, Kapila AK. The impact of amniotic fluid assessed intrapartum an perinatal outcome. Obstet Gynaec Today. 2000;5(8):478-81.

14. Chhabra S, Dargan R. Oligohydramnios - a potential marker for serious obstetric complications. J Obstet Gynecol. 2007;27(7):680-3.

15. Wolff F, Schaefer R. Oligohydramnios-perinatal complications and diseases in mother and child. Geburtshilfe Frauenheilkd Mar. 1994;54(3):139-43.

16. Browen CJS, Kulkarni SK. Amniotic fluid index in the management of postdates pregnancy. West Indian Med J.1995;44(2):61-5.

17. Sarid AL, Levy A. Placental pathologies associated with fetal groth restriction associated with or without oligohydramnios. Arcy Gynecol Obstet. 2009;280(4):549-52.

Cite this article as: Moses V, Thakre S. A study of maternal and fetal outcome in third trimester diagnose case of oligohydramnios. Int J Reprod Contracept Obstet Gynecol 2016;5:2944-8. 